\title{
An Eyeglass Frame Retained Nasal Prosthesis after a Near Total Rhinectomy for Basal Cell Carcinoma of the Nose: A Case Report
}

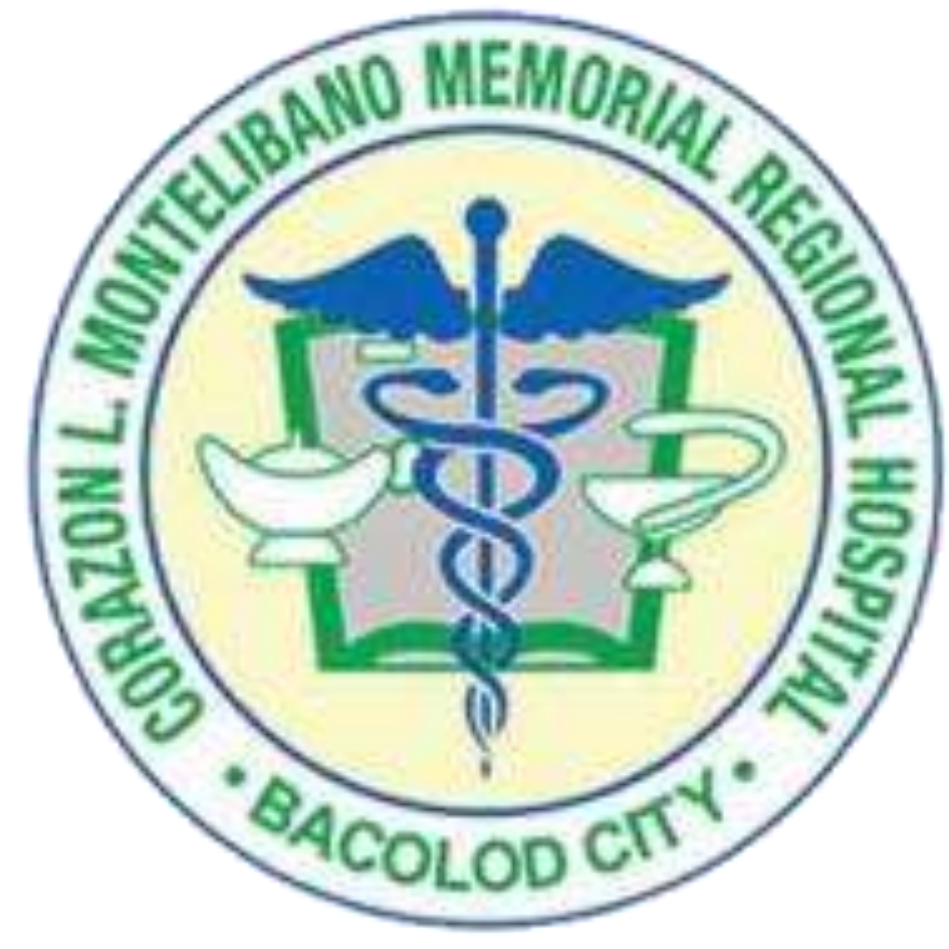

\section{Authors: Josephine Grace C. Rojo, MD, Michael Joseph David, MD Department of Otorhinolaryngology-Head \& Neck Surgery Corazon Locsin Montelibano Memorial Regional Hospital}

\section{Introduction}

Basal cell carcinoma (BCC) is the most common malignancy in humans where $80 \%$ occur in the head and neck region, with almost $25 \%$ appear in the nose. Full thickness involvement can lead to disfiguring surgical defects when resected as seen in this case. Management includes surgical reconstruction techniques, prosthetic rehabilitation, or a combination of both, to address physical and psychological impact of facial disfigurement. However, at present, there is no subspecialty center available within the region offering prosthetic rehabilitation for head and neck patients.

A 64-year old male with BCC of the nose involving about $80 \%$ of the entire nasal area sparing only the right ala and superior nasal bridge (Fig.1) underwent near total rhinectomy with negative surgical margins (Fig. 2).

The following sequential steps were done in the fabrication of nasal prosthesis to address the mid-facial deformity after rhinectomy:

1. Placing of wet gauze as a nasal packing prior to production of an impression of the nasal defect using alginate dental impression material. (Fig. 3, 4)

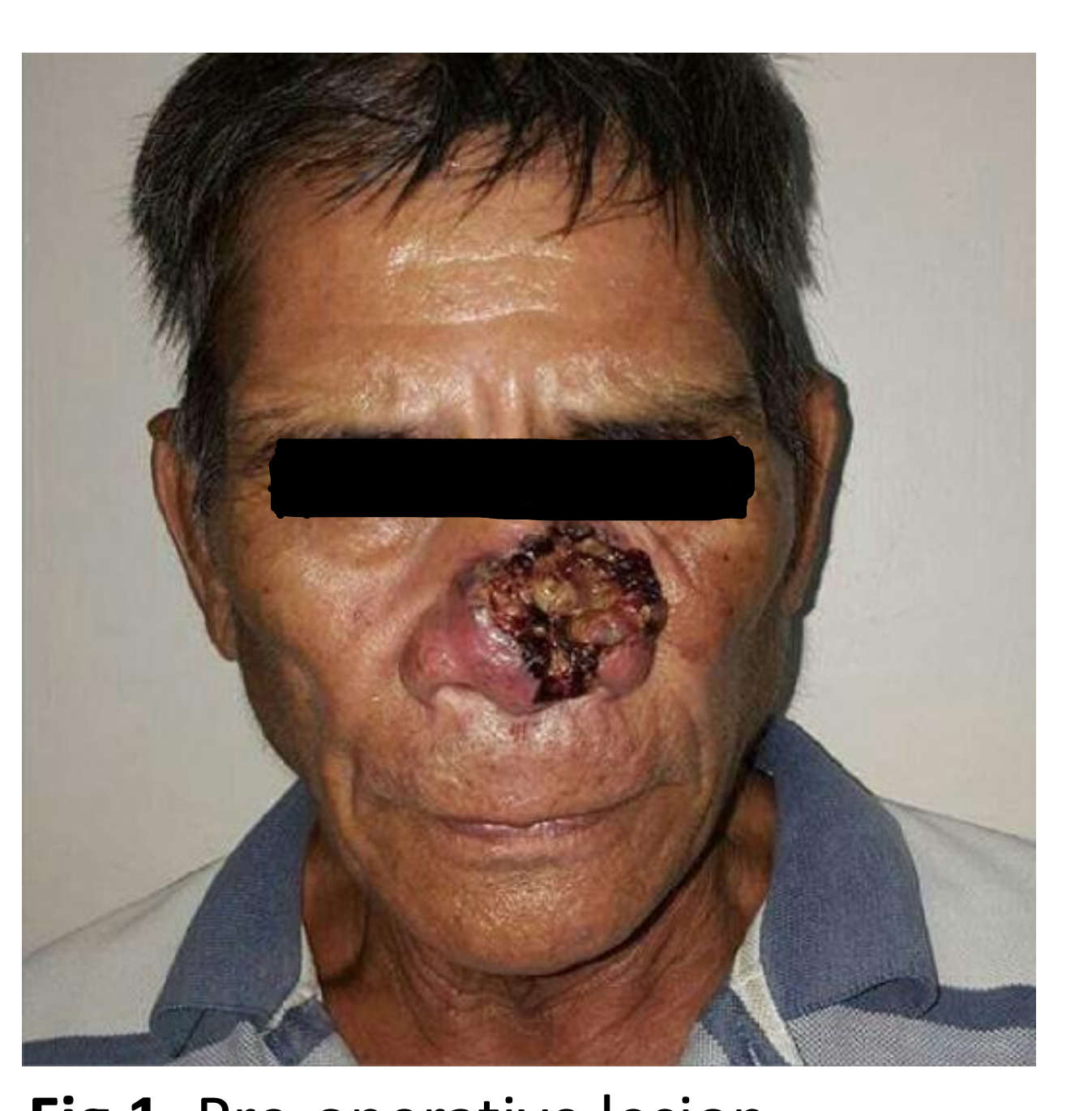

Fig 1. Pre-operative lesion involving most part of nose with extension to nasomaxillary area on

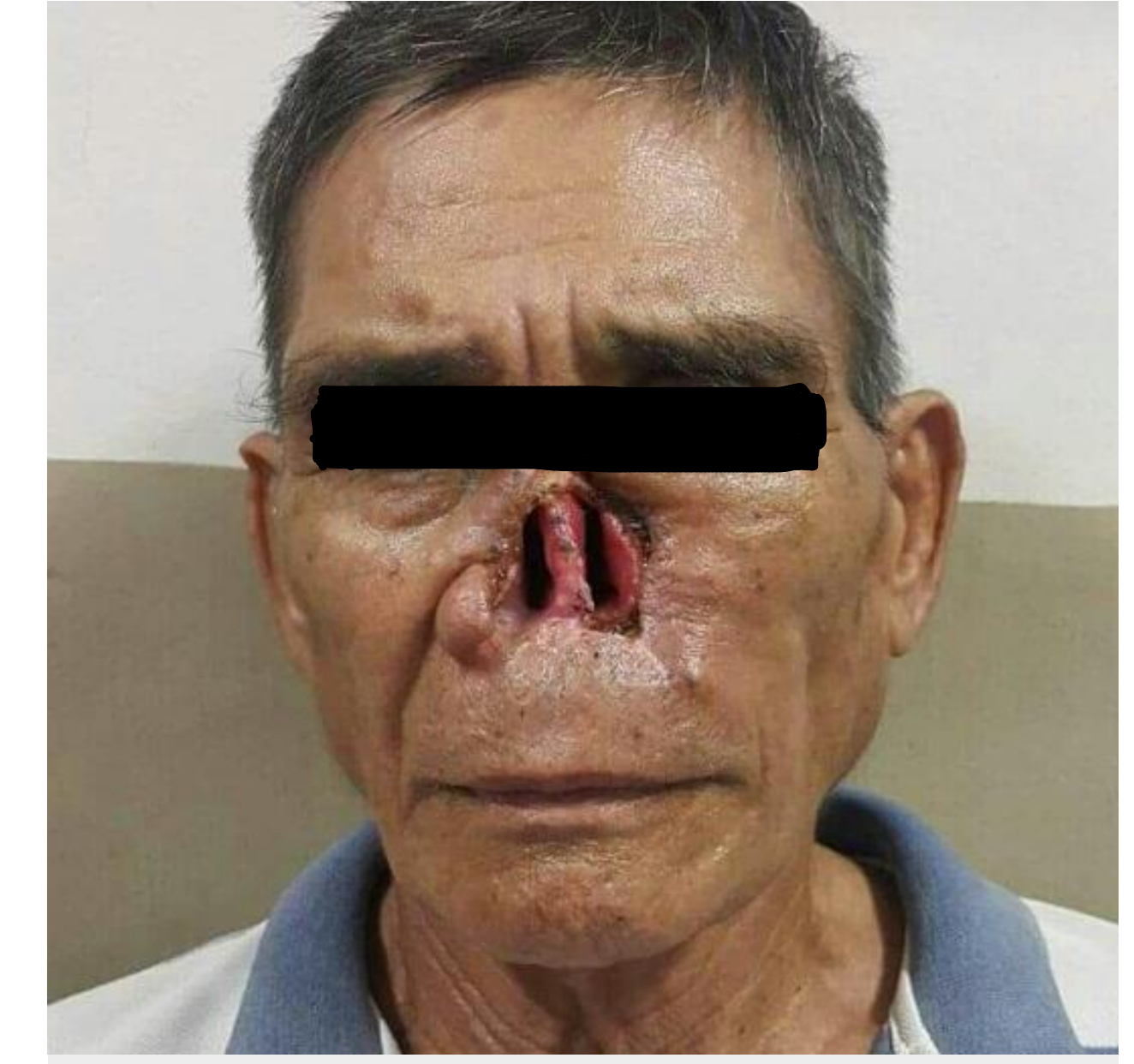

Fig 2. Three weeks post rhinectomy with right nasal ala spared

2. A cast from the impression was used as a foundation to sculpt the mold. (Fig. 5)

3. A trial wax was used to form the desired shape of nasal prosthesis. (Fig. 6)

4. Self-curing medical grade acrylic was used to sculpt the desired nasal prosthesis and was placed on a patient's photo for as reference. (Fig. 7)

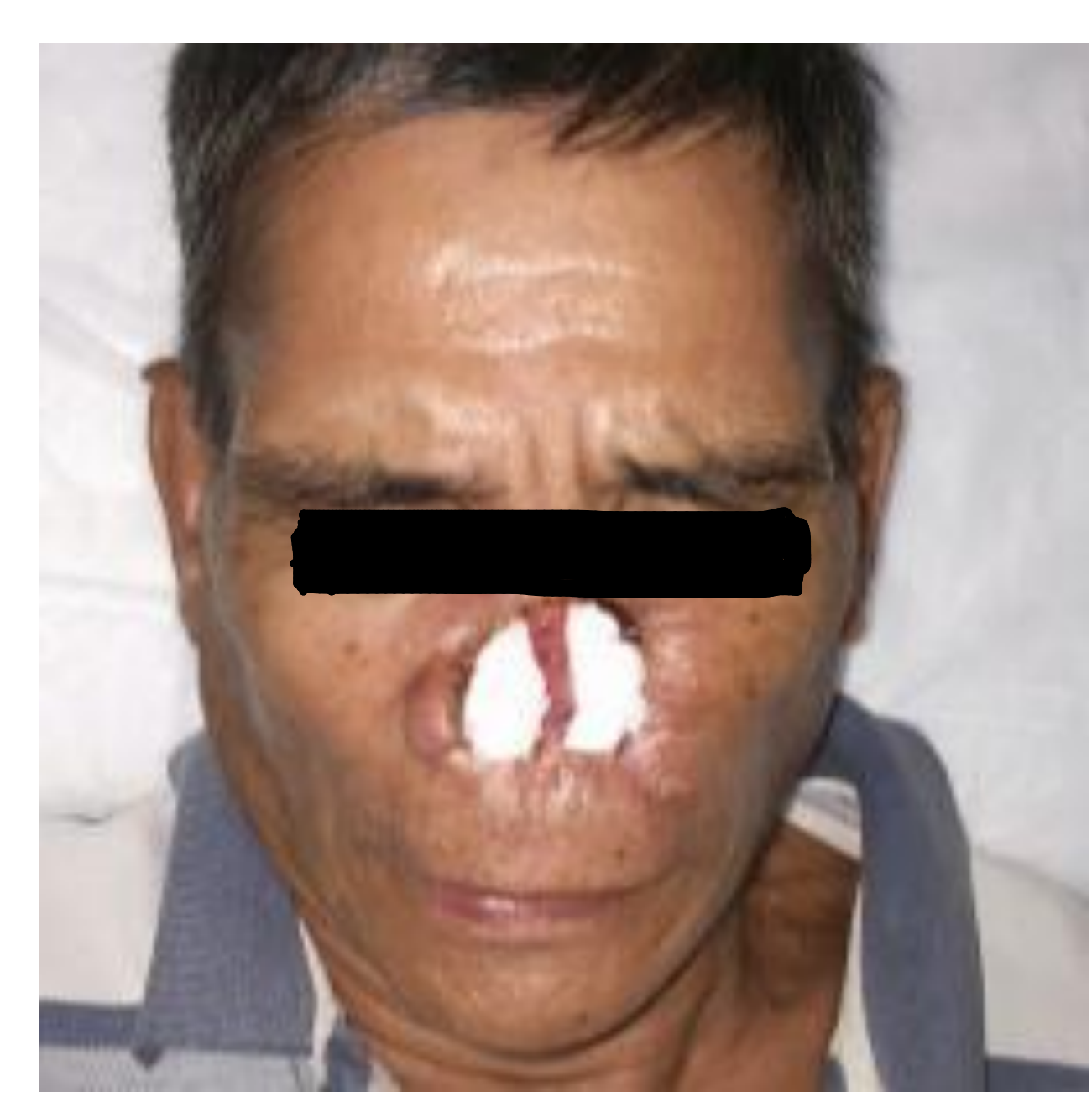

Fig 3. Wet nasal packing soaked in normal saline prior to obtaining an impression using alginate
5. The edges of the nasal prosthesis were smoothened using sand paper.

6 . The nasal prosthesis was painted using nontoxic acrylic paint matching the patient's skin color. (Fig. 8)

7. The nasal prosthesis was attached to an eyeglass frame on the lateral portion of the nasal bridge using industrial grade adhesive. (Fig. 8)
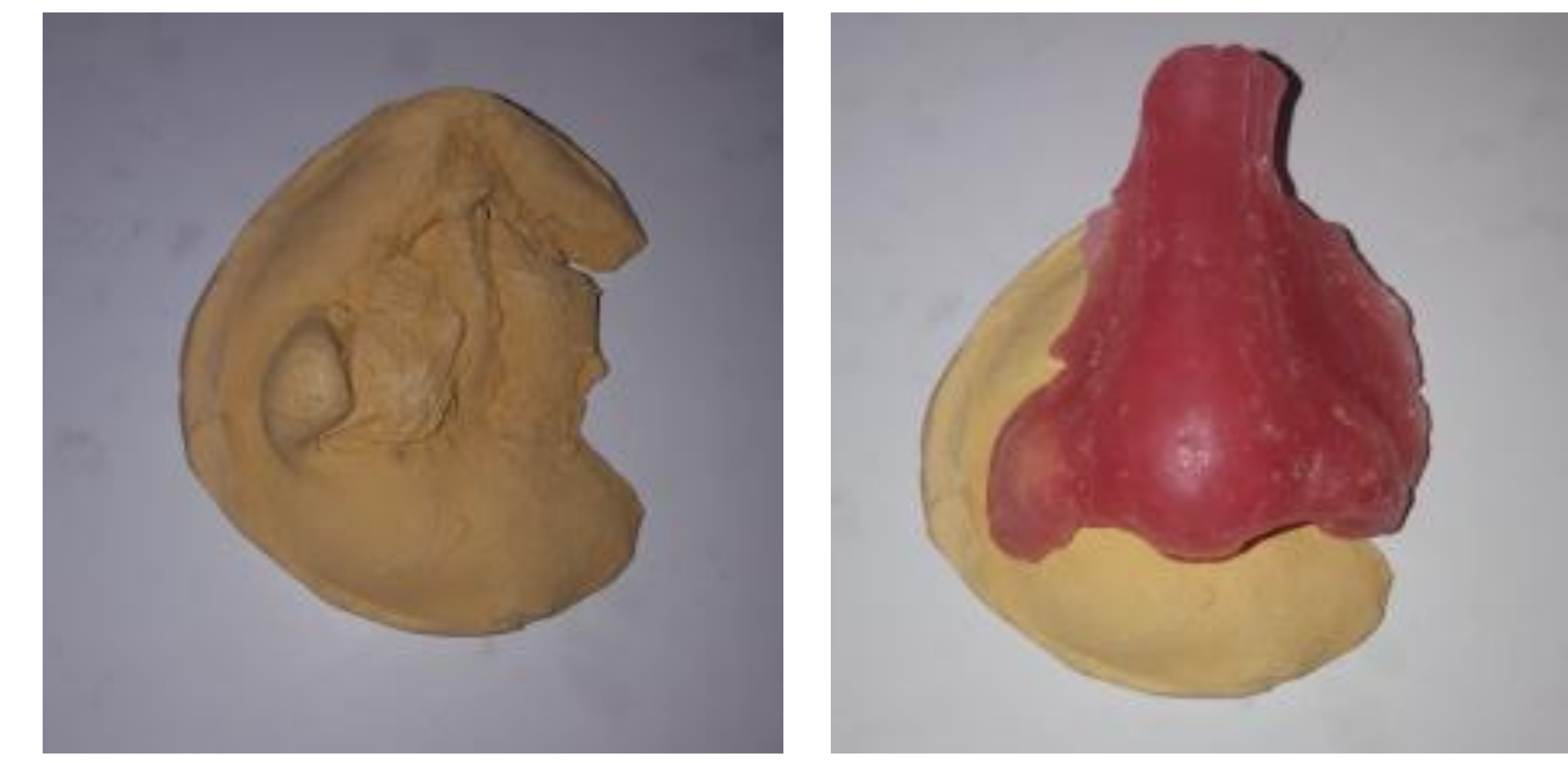

Fig 5. A cast mold of the patient's post- operative site

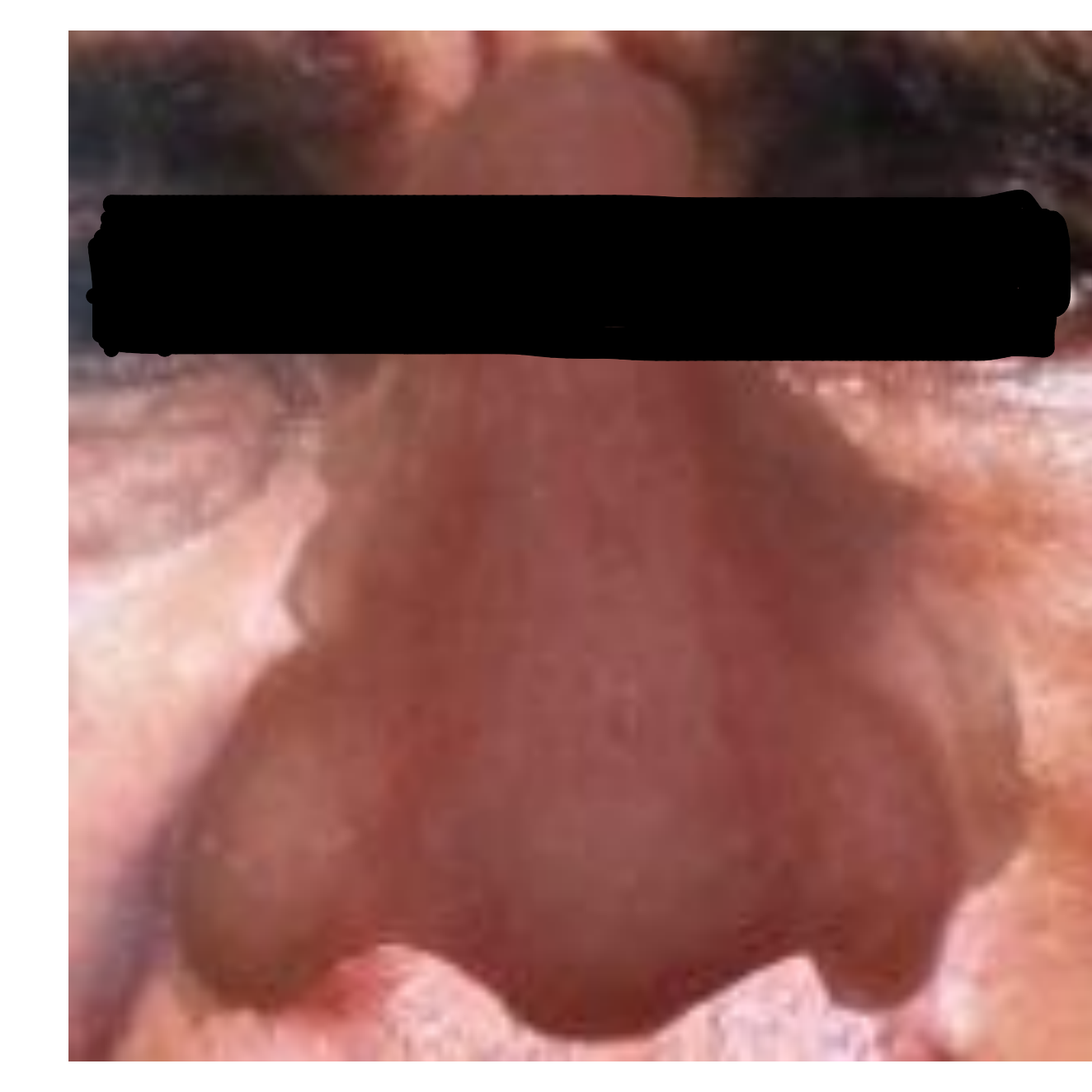

Figure 7. Acrylic nasal prosthetic mold trial on patient's photo
Figure 6. Trial wax on cast mold

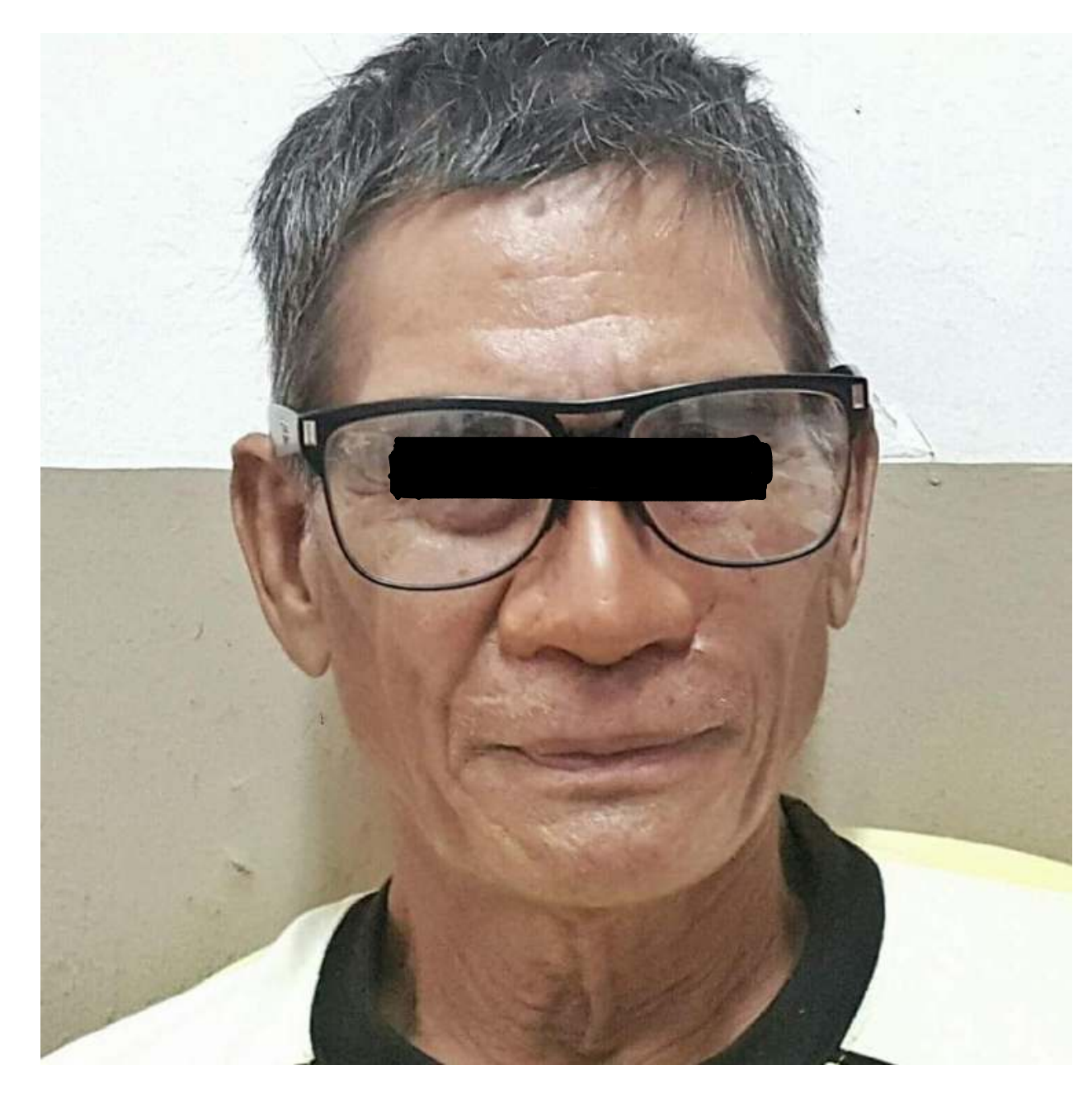

Figure 8. Final eyeglass frame retained nasal prosthesis with acrylic paint on patient using Industrial grade adhesive

\section{Results}

The nasal prosthesis was found to be comfortable and aesthetically acceptable by the patient. Smoothening of the edges of the prosthesis was done to ensure that there are no pressure areas between the prosthesis and mucosa. The weight of nasal prosthesis is supported mostly by the surrounding skin. The cost of nasal prosthesis without the eyeglass frame is approximately Php1,000.00. All materials used are available in most general dental supply stores.

\section{Conclusion}

Facial surgical defects can lead to debilitating post-operative results. However, prostheses can be fabricated to help the patient recover and resume pre-operative activities of daily living with an esthetically acceptable appearance

\section{Reference}

1. Cummings Otolaryngology-Head and Neck Surgery, th $^{\text {th }}$ ed, p 293

2. Lore and Medina an Atlas of Head and Neck Surgery $4^{\text {th }}$ ed., Vol. Lore and

3. Bajaj GB, Shambharkar V. The eyeglass frame retained nasal prosthesis after partial rhinectomy: A clinical report. $\mathrm{I}$. Pakistan Prosthodontic Assoc 2013; 01(02): 113-116. . Gurbuz A, Kalkan M, Ozturk AN, Eskitascioglu G. Nasal Prosthesis Rehabilitation: A Case Report. Quintessence Int. 2004; 35:655-656. 6. Cummings Otolaryngology-Head and Neck Surgery, $6^{\text {th }}$ ed, pp 1417-1418 